\title{
Synthesis of active absorber layer by dip-coating method for perovskite solar cell
}

\begin{abstract}
In this paper, we develop the hybrid perovskite-based n-i-p solar cell using a simple, fast and low-cost dip-coating method. Hot solution and the pre-annealed substrate are used for coating the perovskite thin film by this method this is further used for studying its structural and electrical properties. UV-vis spectroscopy is carried out for calculating the band gap of the hybrid perovskite layer which is $\sim 1.6 \mathrm{eV}$. X-ray spectroscopy confirms that the formation of hybrid perovskite layer. The profilometer is used to study the surface roughness and also for measuring the thickness of the perovskite layer with varying substrate temperature. The optimized sample was further used for cross-sectional SEM image to verify the thickness measured from the profiler. The electrical parameter of JV characteristic with varying temperature is tabulated in the table. Whereas, the perovskite sensitized solar cell exhibits highest short circuit current density, Jsc of $11 \mathrm{~mA} \mathrm{~cm}-2$, open circuit voltage, Voc of $0.87 \mathrm{~V}$, fill factor of 0.55 and efficiency, $\eta$ of $>5 \%$.
\end{abstract}

Keyword: Perovskite sensitized solar cell; Pre-annealing; Dip coating; Mixed-halide perovskite; XRD 